\title{
Change in the ACID Hydrolysis Mechanism of ESTERS ENFORCED by Strongly Acid Microemulsions
}

E. Fernández, L. García-Río, P. Rodríguez-Dafonte

\begin{tabular}{|l|c|}
\hline Stability of AOT and HOT & 2 \\
\hline $\begin{array}{l}\text { Variation of the fraction of neutralized charge }(\beta) \text { with the water content of the } \\
\text { microemulsion in HOT/isooctane/water systems }\end{array}$ & 3 \\
\hline Derivation of Kinetic Equations for Acid Hydrolysis of NPA & 4 \\
\hline Derivation of Kinetic Equations for Acid Hydrolysis of NPL & 8 \\
\hline
\end{tabular}




\section{STABILITY OF AOT AND HoT}

Mass spectra were recorded on a Bruker Microtof ESI-TOF mass spectrometer in highresolution mode using electrospray ionization in the negative mode. The solutions were infused into the ESI source at flow rates of $0.2 \mathrm{~mL} / \mathrm{min}$. We have studied the evolution of the signal $\mathrm{m} / \mathrm{z}=421$ with time for samples of AOT/isooctane/water and HOT/isooctane/water. As can be seen in Figure S-2 there is no variation of the intensities ratio with time indicating that no decomposition is taking place during the interval of time studied.

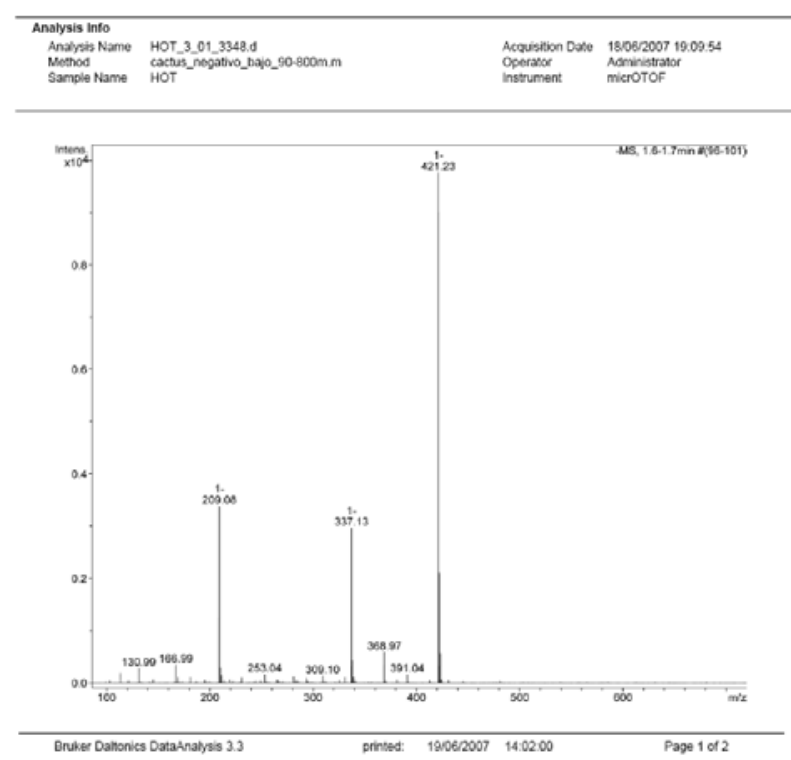

Figure S-1. Mass spectrum of an HOT-based microemulsion. W=20; $[\mathrm{HOT}]=1.00 \mathrm{M}$

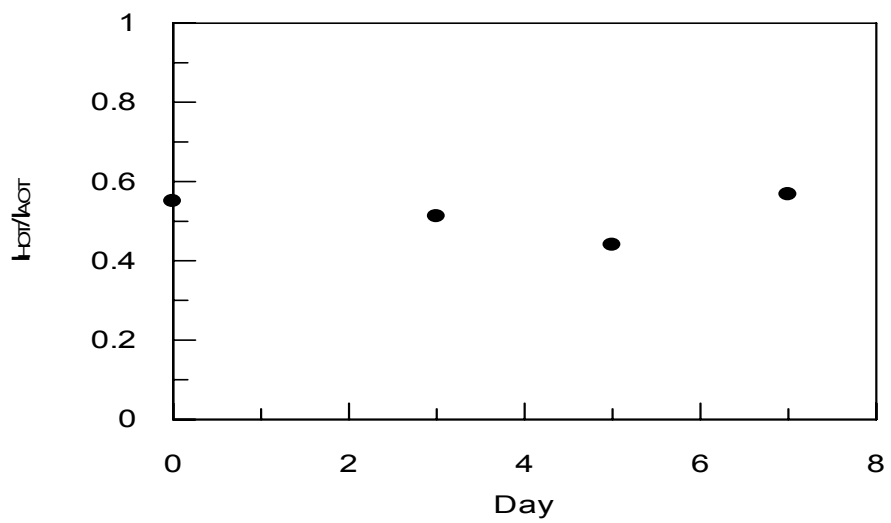

Figure S-2. Variation of the intensities ratio $\left(\mathrm{I}_{\mathrm{HOT}} / \mathrm{I}_{\mathrm{AOT}}\right)$ for the signal with $\mathrm{m} / \mathrm{z}=421$ with time in days. 
Table S-1.Variation of the fraction of neutralized charge $(\beta)$ with the water content of the microemulsion in HOT/isooctane/water systems.

\begin{tabular}{|c|c|}
\hline $\mathbf{W}$ & $\beta$ \\
\hline 1 & 0.928 \\
\hline 2 & 0.928 \\
\hline 3 & 0.928 \\
\hline 4 & 0.928 \\
\hline 5 & 0.929 \\
\hline 6 & 0.930 \\
\hline 7 & 0.931 \\
\hline 8 & 0.931 \\
\hline 9 & 0.931 \\
\hline 10 & 0.930 \\
\hline 11 & 0.930 \\
\hline 12 & 0.930 \\
\hline 13 & 0.929 \\
\hline 14 & 0.929 \\
\hline 15 & 0.929 \\
\hline 16 & 0.928 \\
\hline 17 & 0.928 \\
\hline 18 & 0.928 \\
\hline 19 & 0.928 \\
\hline 20 & 0.927 \\
\hline 21 & 0.927 \\
\hline 22 & 0.926 \\
\hline 23 & 0.926 \\
\hline 24 & 0.926 \\
\hline 25 & 0.925 \\
\hline 26 & 0.925 \\
\hline 27 & 0.925 \\
\hline 28 & 0.924 \\
\hline 29 & 0.924 \\
\hline 30 & 0.924 \\
\hline
\end{tabular}




\section{DERIVATION OF Kinetic EquATIONS FOR ACID HYdRolysis OF NPA}

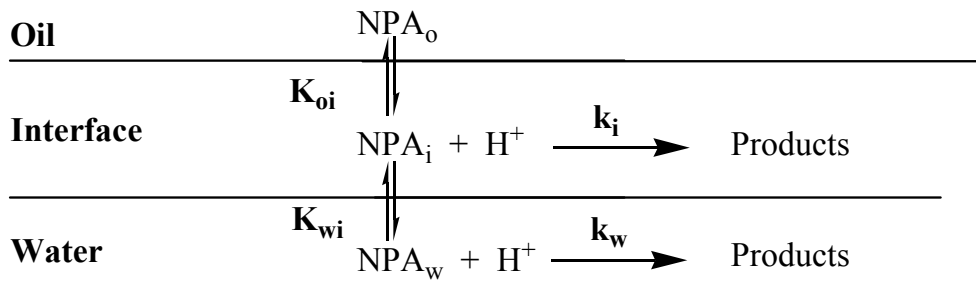

The distribution constant of the NPA throughout the three pseudophases is expressed by means of the following expressions:

$$
\begin{aligned}
K_{o i} & =\frac{[\mathrm{NPA}]_{i}}{[\mathrm{NPA}]_{o}} Z \\
K_{w i} & =\frac{[\mathrm{NPA}]_{i}}{[\mathrm{NPA}]_{w}} W
\end{aligned}
$$

where the concentrations refer to the total volume of the microemulsion and $\mathrm{Z}$ is defined, like W, as Z=[Isooctane]/[HOT]. Using equation (S.1) and (S.2), by means of a simple balance of matter (S.3) we can obtain the following expressions for the NPA concentration in the three pseudophases of the microemulsion:

$$
\begin{aligned}
& {[N P A]_{T}=[N P A]_{o}+[N P A]_{i}+[N P A]_{w}} \\
& {[N P A]_{o}=\frac{K_{w i} Z}{Z K_{w i}+K_{o i} K_{w i}+W K_{o i}}[N P A]_{T}} \\
& {[N P A]_{i}=\frac{K_{o i} K_{w i}}{Z K_{w i}+K_{o i} K_{w i}+W K_{o i}}[N P A]_{T}} \\
& {[N P A]_{w}=\frac{K_{o i} W}{Z K_{w i}+K_{o i} K_{w i}+W K_{o i}}[N P A]_{T}}
\end{aligned}
$$

As shown in Scheme 2, the reagents NPA and $\mathrm{H}^{+}$can come into contact with each other in the aqueous nanodroplet and at the interface of the microemulsion. Therefore, we 
must consider that the reaction takes place simultaneously in water and at the interface so that we can propose the following expression for the rate equation:

$$
\text { rate }=k_{i}^{\prime}[N P A]_{i}+k_{w}^{\prime}[N P A]_{w}
$$

where $k_{i}^{\prime}$ and $k_{w}^{\prime}$ are the rate pseudoconstants of the first order referred to the interface and the aqueous microdroplet, respectively. Substitutiting $[N P A]_{i}$ and $[N P A]_{w}$ in (S.7):

$$
\text { rate }=\left(k_{i}^{\prime} \frac{K_{o i} K_{w i}}{Z K_{w i}+K_{o i} K_{w i}+W K_{o i}}+k_{w}^{\prime} \frac{K_{o i} W}{Z K_{w i}+K_{o i} K_{w i}+W K_{o i}}\right)[N P A]_{T}
$$

Then we can obtain the following expression for the observed rate constant:

$$
k_{o b s}=\frac{k_{i}^{\prime} K_{o i} K_{w i}+k_{w}^{\prime} K_{o i} W}{Z K_{w i}+K_{o i} K_{w i}+W K_{o i}}
$$

The rate pseudoconstants $k_{i}^{\prime}$ and $k_{w}^{\prime}$ can be expressed as bimolecular rate constants at the interface, $k_{i}$, and the aqueous microdroplet, $k_{w}$, as:

$$
\begin{aligned}
& k_{i}^{\prime}=k_{i}\left[H^{+}\right]_{i}^{i} \\
& k_{w}^{\prime}=k_{w}\left[H^{+}\right]_{w}^{w}
\end{aligned}
$$

where $\left[\mathrm{H}^{+}\right]_{i}^{i}$ and $\left[\mathrm{H}^{+}\right]_{w}^{w}$ refer to the $H^{+}$concentration at the interface and the aqueous microdroplet of the microemulsion referred to the volume of the interface and the aqueous phase respectively. These concentrations are transformed into concentrations referred to the total volume of the system by taking into account just the phase volumes:

$$
\begin{gathered}
{\left[H^{+}\right]_{i}^{i}=\frac{\left(n_{H^{+}}\right)_{i}}{V_{i}}=\frac{\left(n_{H^{+}}\right)_{i}}{\bar{V}_{H O T} \cdot n_{H O T}} \frac{V_{T}}{V_{T}}} \\
{\left[H^{+}\right]_{w}^{w}=\frac{\left(n_{H^{+}}\right)_{w}}{V_{\mathrm{H}_{2} \mathrm{O}}}=\frac{\left(n_{\mathrm{H}^{+}}\right)_{w}}{\bar{V}_{\mathrm{H}_{2} \mathrm{O}} \cdot n_{\mathrm{H}_{2} \mathrm{O}}} \frac{V_{T}}{V_{T}}}
\end{gathered}
$$


Taking into account:

$$
\begin{aligned}
& \left.\begin{array}{l}
\frac{\left(n_{H^{+}}\right)_{i}}{V_{T}}=\left[H^{+}\right]_{i} \\
\frac{n_{H O T}}{V_{T}}=[H O T] \\
\frac{\left(n_{H^{+}}\right)_{w}}{V_{T}}=\left[H^{+}\right]_{w} \\
\frac{n_{H_{2} O}}{V_{T}}=\left[H_{2} O\right] \\
W=\frac{\left[H_{2} O\right]}{[H O T]}
\end{array}\right\} \quad\left[H^{+}\right]_{i}^{i}=\frac{\left[H^{+}\right]_{i}}{\bar{V}_{H O T}[H O T]} \\
& {\left[H^{+}\right]_{w}^{w}=\frac{\left[H^{+}\right]_{w}}{\bar{V}_{H_{2} O} W[H O T]}} \\
&
\end{aligned}
$$

And replacing the expressions (S.14) and (S.15) in the equation (S.12) and (S.13) we obtain:

$$
\begin{aligned}
& k_{i}^{\prime}=k_{i} \frac{\left[H^{+}\right]_{i}}{\overline{\mathrm{V}}_{\text {НОт }}[\mathrm{HOT}]} \\
& k_{w}^{\prime}=k_{w} \frac{\left[H^{+}\right]_{w}}{W \bar{V}_{\mathrm{H}_{2} \mathrm{O}}[\mathrm{HOT}]}
\end{aligned}
$$

$\left[\mathrm{H}^{+}\right]_{i}$ and $\left[\mathrm{H}^{+}\right]_{w}$ correspond to the $\mathrm{H}^{+}$concentration at the interface and the aqueous microdroplet of the microemulsion referred to the total volume of the system.

As is usual in microemulsions and aqueous micelles, the interface has been considered to be consisting solely of the surfactant, in such a way that the volume of the interface is given by the volume occupied by the surfactant. In this way $V_{t o t} / V=1 / \bar{V}_{\text {HOT }}[H O T]$ where $\bar{V}_{H O T}=0.418 \mathrm{M}^{-1}$ and $[\mathrm{HOT}]$ represents the total concentration of HOT referred to the total volume of the microemulsion. We can express the $H^{+}$concentration at the interface according to the fraction of the neutralized micellar charge, $\beta$ :

$$
\beta=\frac{\left[H^{+}\right]_{i}}{[H O T]}
$$


We can rewrite equations (S.16) and (S.17):

$$
\begin{aligned}
& k_{i}^{\prime}=k_{i} \frac{1}{\overline{\mathrm{V}}_{\mathrm{HOT}}} \beta \\
& k_{w}^{\prime}=k_{w} \frac{1}{W \bar{V}_{\mathrm{H}_{2} \mathrm{O}}}(1-\beta)
\end{aligned}
$$

By replacing the expressions (S.19) and (S.20) in the equation (S.9) we obtain:

$$
k_{o b s}=\frac{k_{i} \frac{\beta}{\bar{V}_{H O T}}+k_{w} \frac{1-\beta}{K_{w i} \bar{V}_{H_{2} O}}}{1+\frac{W}{K_{w i}}+\frac{Z}{K_{o i}}}
$$

The inverse of the equation (S.21) predicts the existence of a linear relation between $1 / k_{\text {obs }}$ and the $\mathrm{Z}$ parameter of the microemulsion composition.

$$
\frac{1}{k_{o b s}}=\frac{\bar{V}_{H O T} \bar{V}_{H_{2} O}\left(K_{w i}+W\right)}{K_{w i} \beta \bar{V}_{H_{2} O} k_{i}+(1-\beta) \bar{V}_{H O T} k_{w}}+\frac{\bar{V}_{H O T} \bar{V}_{H_{2} O} \frac{K_{w i}}{K_{o i}}}{K_{w i} \beta \bar{V}_{H_{2} O} k_{i}+(1-\beta) \bar{V}_{H O T} k_{w}} Z
$$




\section{DERIVATION OF KINETIC EQUATIONS FOR ACID HYDROLYSIS OF NPL}

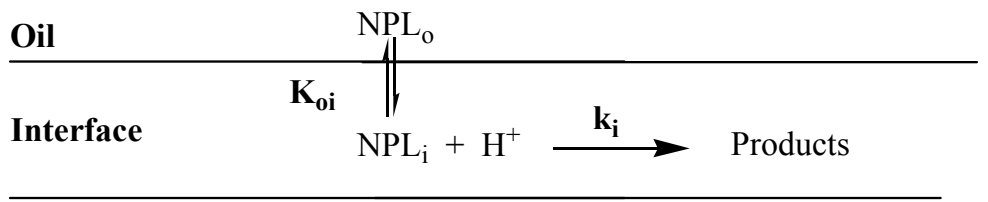

Water

The reaction takes place only at the interface so that we can propose the following expression for the rate equation:

$$
\text { rate }=k_{i}^{\prime}[\mathrm{NPA}]_{i}
$$

The distribution constant of the NPL:

$$
K_{o i}=\frac{[\mathrm{NPL}]_{i}}{[\mathrm{NPL}]_{o}} Z
$$

The NPL concentration in the two pseudophases:

$$
\begin{aligned}
& {[\mathrm{NPL}]_{i}=\frac{K_{o i}}{K_{o i}+Z}[\mathrm{NPL}]_{T}} \\
& {[\mathrm{NPL}]_{o}=\frac{Z}{K_{o i}+Z}[\mathrm{NPL}]_{T}}
\end{aligned}
$$

Equation (S.23) can be rewritten:

$$
\text { rate }=k_{i}^{\prime} \frac{K_{o i}}{K_{o i}+Z}[N P L]_{T}
$$

The observed rate constant is: 


$$
k_{o b s}=k_{i}^{\prime} \frac{K_{o i}}{K_{o i}+Z}
$$

The rate pseudoconstant $k_{i}^{\prime}$ can be expressed as bimolecular rate constants at the interface, $k_{i}$ :

$$
k_{i}^{\prime}=k_{i}\left[\mathrm{H}^{+}\right]_{i}^{i}=k_{i} \frac{\mathrm{V}_{\mathrm{T}}}{\mathrm{V}_{\mathrm{i}}}\left[\mathrm{H}^{+}\right]_{i}=k_{i} \frac{1}{\overline{\mathrm{V}}_{\mathrm{HOT}}} \beta
$$

Finally:

$$
k_{\text {obs }}=k_{i} \frac{\beta}{\overline{\mathrm{V}}_{\text {НОТ }}} \frac{K_{o i}}{K_{o i}+Z}
$$

